\title{
Using Social Media During the Hiring Process: A Comparison Between Recruiters and Job Seekers
}

\author{
Elizabeth C. Alexander, alexanec@marshall.edu* \\ Deanna R. D. Mader, maderd@marshall.edu \\ Fred H. Mader, mader@marshall.edu
}

\section{Introduction}

Jobvite, a recruiting platform for the social web, reports from their annual 2012 survey of recruiters that $92 \%$ of U.S. companies are using social networking sites (SNS) for hiring purposes (Jobvite 2012). CareerBuilder reported in 2009 that $45 \%$ of employers were using SNS to screen and research applicants (CareerBuilder 2009). That number has increased to 59\% as of the most recent survey on 2016 (Career Builder 2016). According to the Society for Human Resource Management, 77\% of its members surveyed in 2013 report using social media for recruiting purposes (Segal 2017). It is important that faculty and support staff working to place students, and the students themselves, understand the developments and practices in the use of social networking sites for job search and recruiting and the best methods, as well as detriments, when marketing themselves. This study compares corporate recruiters', operating on a college campus, attitudes toward the use of social media in recruiting with students' (job seeker) attitudes.

While work has started in understanding the uses of SNS for recruiting, additional research is necessary to fully understand who is using SNS for hiring decisions and how those decisions are made in light of the SNS content being evaluated, as well as the use of social media by job seekers. It is important to determine if the evaluations of social media by these two groups are somewhat similar in nature. Otherwise, social media use and content by the job seeker may be deemed inappropriate or not in fitting with the organization and/or position by a recruiter screening applicants via social media. Soon-to-be and recent graduates should be made aware of the types of content which may impact their success rate in finding a position.

\section{Previous Research}

Using social networking sites like Facebook, Twitter and LinkedIn is becoming more popular with both job seekers and employers. This research seeks to understand recruiters' evaluation and behavior with regard to utilizing SNS for hiring decisions. As Strehlke (2010) points out, the use of SNS can improve the visibility of job seekers, but not without its challenges. The conclusion, after a review of the available literature, is for attention to privacy and work/personal life issues, online presentation, managing online information and networking practices, as well as potential legal challenges, especially when SNS use uncovers areas of the applicant that are not relevant to the job (Black \& Johnson 2012).

While the reported percentages seem to show an increasing use of SNS for hiring purposes, research in the area is lacking as suggested by Brown and Vaughn (2011), Davison, Maraist and Bing (2011) and Black and Johnson (2012). Very little empirical research exists in the areas of utilizing SNS for either the recruiting or screening processes. One potential benefit of using SNS for hiring procedures involves corroboration of applicant provided information from other sources, where recruiters "associate transparency with screening job applicants on online social networks" (El Ouirdi, Pais, Segers, \& El Ouirdi 2016), such as the application or resume. The cost to uncover potentially valuable information is very low relative to other methods of obtaining the same information. Recruiters using SNS are able to get a feel for the candidates' "fit" with the organizations, as well as to evaluate their communication skills and creativity. They can also use the SNS information as supporting evidence of qualifications listed in other hiring materials, i.e. resume or application (Jones \& Behling 2010, El Ouirdi et al. 2016). Potential risks have also been discussed. Invasion of privacy, whether perceived or actual, is a potential risk. The information obtained through the SNS search could suffer from a lack of job relevance. The information from SNS is also, often, evaluated without complete, or any, contextual background. Discrimination, blatant or otherwise, may also occur (Brown \& Vaughn 2011). Black and Johnson (2012) propose that, with the use of SNS, discrimination would exist for a variety of reasons including the underrepresentation of minorities on job sites, a more favorable perception of applicants with a social media site than those without, and also a more 
favorable perception of men and younger applicants.

Employers using social media to evaluate job candidates reject applicants due to a variety of issues uncovered through a social media search (CareerBuilder 2014). The most common reason being inappropriate photos (46\%). Other reasons include drinking or drug use, negative comments about current or past employer, poor communication skills, offensive or discriminatory comments, qualifications not substantiated, and criminal behavior. Twenty-one percent have even passed on a candidate for an unprofessional screen name. While there seem to be a plethora of reasons to reject, $33 \%$ of the employers using social media to research a candidate do report hiring due to content found on social media. Favorable content includes a good personality/company fit, professional image, good communication skills, and creativity. Recruiters also liked when the candidate interacted with the organization's social media accounts.

Davison (2012) organizes the type of search, the sources visited, into 4 levels of risk. Level 1 and 2 represent the least risk and mild risk, respectively. In these cases the recruiter is searching for information on sites either referenced by the applicant or previous employment sites owned by the company or the applicant themselves. These searches usually result in substantiating applicant provided information. Level 3 and 4 search involve searching the web for postings on social network sites by the applicant with Level 4 looking at 3rd party postings concerning the applicant. Davison points out that using information from level $3 \& 4$ searches increases the chance of finding irrelevant information about the candidate which, if used in the selection process, would leave the employer in a precarious position legally.

Segal (2017) reports that several employers, 74\% in a 2013 SHRM survey, mention legal risks when determining whether to use a candidate's social media profiles. As Segal mentions, existing regulations and laws are useful in determining the boundaries of utilizing social media to screen applicants and the human resource department would be best equipped to perform those searches. Lamoureux (2012) noted that as SNS become a more common way for college students and companies to connect, there will be significant legal issues concerning privacy and discrimination.

In addition, Lamoreux suggested that employers must understand that SNS case law will be in infancy for some time and that the boundaries for what is acceptable are still to be formed.

A few studies have examined the use of SNS by either recruiters or job seekers as noted by El Ouirdi, et al. (2016). One study (Nikolaou 2014) examined the use of Facebook, LinkedIn and job boards by both job seekers and human resource professionals. In general, findings indicate that younger job seekers and human resource professionals tend towards Facebook and job boards, while the older in both groups utilize LinkedIn. A higher education level is associated more with LinkedIn for the job seeker. Males also tended to use LinkedIn more than females for both the job seeker and human resources professionals groups. Job boards were still seen as an effective tool for job seekers.

Chang and Madera (2012) examined the use of social network sites for selection in the hospitality industry. In a small sample, using dichotomous response formats, the research found that $54 \%$ of the respondents reported their companies use social networking sites to screen applicants but only $18.5 \%$ revealed there is a company policy regarding the use of social media for recruiting purposes. More important for applicants, the authors found that negative information was viewed as more important than positive information found on the applicant's site, validating the positive-negative asymmetry effect.

Only a handful of studies have investigated the impact of SNS postings and recruitment evaluations. When respondents were asked to evaluate "applicants" via SNS with the presence or absence of the attributes such as a professional resume, an emphasis on drinking and family orientation, they ranked the applicants with "unprofessional "websites and an emphasis on drinking as less attractive and would offer them a lower salary, if hired (Bohnhert \& Ross 2010). Alcohol, along with gambling, presented as information during a hiring process 
was also the focus of a study (Weathington \& Bechtel 2012). Participants were to evaluate a candidate based on a resume and the candidate's personal web pages. The presence of alcohol consumption negatively impacted the 5 areas assessed for recommending an interview with the applicant: qualifications, likelihood of a hiring recommendation, expected performance and likelihood of candidate quitting. In contrast, gambling was only significant in the assessment of the likelihood of the candidate quitting.

Goodmon, et al. (2014) conducted a study to determine how SNS content, specifically Facebook content, influences the ability to evaluate personality. Participants in this study were exposed to three types of content: professional, moderately-professional and unprofessional from three actual Facebook users who modified their pages to reflect the three levels of professional content. Each varied the amount of inappropriate content. Evaluations were then made on the Big Five Personality characteristics and the potential of hiring. Results show a significant effect on the personality dimension. Differences based on the level of professionalism were also found between the respondents' ratings and the self-ratings of the three individuals portrayed in the Facebook profiles. Interestingly, willingness to hire was not affected. In an earlier, similar study, participants were able to accurately determine high and low scorers in the Big 5 personality dimensions from actual, non-manipulated Facebook profiles, when compared to the self-reported or true scores (Kluemper \& Rosen 2009).

Participants, in a study regarding faux pas postings (Roulin 2014), were placed in an experimental condition which manipulated the proportion of employers using SNS to evaluate candidates (high versus low) and the degree of privacy invasions by employers (no information on invasion versus information on invasion), and were then measured on the likelihood of engaging in self-promotion behaviors online as well as the likelihood of utilizing ten types of faux pas postings. A faux pas posting is one that may hinder the individual's chances of getting a job, such as posting pictures related to alcohol consumption or drug use. Results show that participants were likely to alter their social media profile when it was known that recruiters actively sought information from the SNS, to "adapt their behavior to match employers' selection criteria and strategies."

El Ouirdi et al. (2016) examined recruiter gender and nationality (specifically the Netherlands and Italy) effects when assessing candidate postings, nonprofessional and professional. According to their study, these two cultures rely on different levels of context in communications. The Dutch are classified as a low-context, rule-based culture, that utilizes frankness and logic when communicating. Italians are classified as high-context, meaning that, in their relationship-based culture, networking and pre-established relationships provide much of the information reducing background check and screening requirements. These two cultures also differ when selecting applicants. The Dutch preferring "hard criteria" and the Italians relying on social class and other "soft criteria." Comparing gender and nationality of recruiters, this study found no significant difference when assessing nonprofessional content between genders, but Dutch recruiters did assess non-professional content significantly more negative than did Italian recruiters. Also, non-professional assessment was linked to eliminating the candidate from consideration (El Ouirdi, et al. 2016).

Several studies have also examined the use of social media by job seekers and evaluations of its benefits. In an early contribution, Petrecca (2011) suggested that SNS was not just being used by employers to screen applicants, but was also being used by applicants to peruse potential employers. Petrecca suggested that companies may need to revamp their SNS materials in order to attract top notch college graduates. Accounting students, surveyed by Herbold and Douma (2013) were found to use few social networking sites in the job search process, less than one fifth, with 19.3\%, using online sites for any reason during the search process. The authors found that users of social networking sites differed from non-users by the following characteristics: they were slightly older, likely to be graduate students and heavier users of social media. The users, for the most part, engaged in social media during the earlier stages of the job search process, using the resource to research the organization and conduct searches for jobs and internships. Users reported more proactive activity on social media than non-users by evaluating their own social media profiles, posting resumes online and joining online networks. When asked to rate the usefulness of social media for recruiting on a 5-pt scale where $1=$ not at all useful and $5=$ very useful, both users and non-users were less than convinced of the benefits of using social media for job search rating 
usefulness 2.44 and 1.70 , respectively.

A few studies have examined the degree of awareness job seekers have of the use of SNS by recruiters and/or what actions job seekers take in response to that awareness. Root and McKay (2014) surveyed college of business students. They found that students overwhelmingly $(80 \%)$ believe that prospective employers are "likely" or "very likely" to check the Facebook profiles of applicants. Although students did report awareness of faux pas such as including drug-related information, sexual or racial comments, alcohol use, inappropriate pictures, and disparaging comments about employers; they did not report awareness of the need for proper spelling, grammar, and general communication issues. Williams, Feild, and James (2011) examined how PharmD students change their information sharing on Facebook once they become aware of the college's social media policy. The authors found that students who had fewer security settings strengthened settings once they became aware of the college's social media policy suggesting that once students are aware that their SNS materials are being monitored, they will take action to manage the impression created for specific social groups. French and Read (2013) explored how the depth of information shared on Facebook changes based on the audience (social spheres) having access to the material. The authors noted that impression management theory could explain why depth of information sharing is reduced as social spheres move from friends, to family, and then to co-workers.

\section{Methodology}

In the current research, an electronic survey was administered to recruiters registered through a campus career services department. Items measured included social media behavior and evaluations of particular types of social media content (religious, drugs, sexual, etc.). Recruiters were also asked about the usefulness of social media in choosing applicants and the uses of social media in recruiting, along with basic demographic variables. The electronic distribution yielded 202 useable surveys.

A similar instrument was used to access those who would be a job seeker, and resulted in 441 responses representing college students and members of the general population. In addition to social media behavior and evaluations of types of social media content, these respondents were also asked to report on the likelihood of using social media in various ways during job search. The job seekers were asked to respond to the same items as the recruiters regarding the usefulness of social media in choosing applicants and the uses of social media in recruiting.

\section{Hypotheses}

Individuals post various activities and opinions on their social media platforms. It is expected that these different types of content will have different levels of acceptance among the recruiters and job seekers and that these two groups will rate the content slightly differently. For example, illegal activities should garner a more negative response than profanity for both recruiters and job seekers. It is expected, that in general, the less socially acceptable behaviors will be rated as more negative than the more acceptable behaviors, such as volunteerism. Recruiters may also rate activities that can be used to evaluate a candidate more highly than job seekers. By evaluating the differences between recruiters and job seekers, recommendations can be made to the job seeker concerning what to post and not post while on the job market.

H1a: The mean for negative social media content will be less for recruiters than for job seekers, indicating a more negative impression.

H1b: The mean for positive social media content will be higher for recruiters than for job seekers, indicating a more positive impression of that type of content.

Recruiters and job seekers were also asked their level of agreement with several statements regarding the usefulness of SNS sites for evaluating potential employees and how far a recruiter could go when utilizing SNS.

It is expected that there will be differences between these two groups. Job seekers may not expect or feel that their social media pages would or should be used for hiring purposes. 
H2a: Recruiters will have a higher level of agreement that social media actions should be used in the hiring process to screen applicants than job applicants.

$\mathrm{H} 2 \mathrm{~b}$ : Recruiters will have a higher level of agreement that social media is a good tool in screening applicants than job applicants.

\section{Results}

Recruiters: Respondents to the recruiter instrument represented a variety of industries, from local to international and with less than 100 to over 1000 employees. A majority of the individual respondents had experience in using social media personally; 161 had at least one social media account and 118 of those signed into their account at least once a day. Both male and female recruiters were represented with a wide range of ages. All but four of the respondents had more than a high school education.

Out of the 202 useable responses, 162 of the organizations represented had at least 1 social media account. The most used social media account was Facebook with 152 organizations represented. Other social media platforms used were Twitter, LinkedIn and Google+, with 91, 85 and 29 organizations using. Instagram, Pinterest, MySpace and SecondLife were also represented. The organizations responded that all the platforms, except Pinterest, were used to advertise job positions, with Facebook being used the most (68), followed by LinkedIn (58).

When asked about their use of social media to evaluate job applicants, overall, these organizations were only moderately active. Only 31 replied that the organization searches for every applicant's social media profile; whereas 44 search, but not for every applicant. A total of 112 responded in the categories of searching Not very often or Not at all.

Job Seekers: Of the 441 responses to the survey directed towards individuals in or likely to be in the job market in the future, 91\% reported having at least one social media account, with the majority reporting that Facebook was their main site. A large portion of the respondents maintained accounts on Facebook (348), while smaller numbers reported maintaining accounts on Twitter (196), Pinterest (146), Instagram (106), LinkedIn (89), Google (52), MySpace (28) and SecondLife (6).

One-third of the respondents signed into their account and kept it open all day and only $18 \%$ reported signing in less than once a day. About half of the respondents (223) spent less than 2 hours per day engaged in any social media, 110 spent $2-4$ hours per day, and 30 respondents replied they spent more than 4 hours per day engaged.

Comparing Recruiter and Job Seeker Positions: This research seeks to determine if there is a gap between the recruiters' and job seekers' ratings on social media activities, content and usefulness. In most cases, there were significant differences (see Table 1). Job seekers were less negative when rating content types such as illegal drugs and other questionable types of content and less positive when rating content that could be beneficial to the job search. For example, profanity, sexual content, illegal drug use and pictures with alcohol were rated more negatively by recruiters than by job seekers, while acceptable types of content, e.g. religious content, professional organization membership and volunteer activities, were rated more positively by recruiters. Hypothesis $1 \mathrm{a}$ is supported with the exception of the rating for Spelling/Grammar Mistakes. Interestingly, job seekers rated spelling grammar mistakes significantly more negative than the recruiters', 1.89 and 2.10, respectively.

Hypothesis $1 \mathrm{~b}$ is partially supported. Recruiters ranked positive social media content more favorably than job applicants with significant differences occurring with the means of Professional Organization Membership and Volunteer/Donation activities. Religious content was rated higher by recruiters, but not significantly so. See Table 1 for all means and significance values. 
Table 1

\begin{tabular}{|l|l|l|l|}
\hline Content Type & $\begin{array}{l}\text { Job Seeker } \\
\text { Mean }\end{array}$ & $\begin{array}{l}\text { Recruiter } \\
\text { Mean }\end{array}$ & Sig. \\
\hline Illegal Drugs & 1.41 & 1.12 & .000 \\
\hline Sexual Content & 1.68 & 1.51 & .002 \\
\hline Spelling/Grammar Mistakes & 1.89 & 2.10 & .000 \\
\hline Profanity & 2.00 & 1.69 & .000 \\
\hline Pictures with Alcohol & 2.21 & 2.12 & .104 \\
\hline Religious Content & 2.98 & 3.02 & .589 \\
\hline $\begin{array}{l}\text { Professional Organization } \\
\text { Membership }\end{array}$ & 3.76 & 4.06 & .000 \\
\hline Volunteer/Donation Activities & 3.86 & 4.29 & .000 \\
\hline
\end{tabular}

$1=$ Very Negative and $5=$ Very Positive

When rating the appropriateness of certain recruiter activities on social media, again, differences occur between recruiters and job seekers. Job seekers were less accepting of those activities than recruiters. All item means were found to be significantly different; thus, Hypotheses $2 \mathrm{a}$ and $2 \mathrm{~b}$ are both supported (see Table 2 ).

Table 2

\begin{tabular}{|l|l|l|l|}
\hline Activity & $\begin{array}{l}\text { Job Seeker } \\
\text { Mean }\end{array}$ & $\begin{array}{l}\text { Recruiter } \\
\text { Mean }\end{array}$ & Sig. \\
\hline $\begin{array}{l}\text { Potential Employers should search social media for } \\
\text { applicant information }\end{array}$ & 2.93 & 2.38 & .000 \\
\hline $\begin{array}{l}\text { Applicants should be able to deny social media } \\
\text { access to employers without negative effects }\end{array}$ & 1.95 & 2.53 & .000 \\
\hline $\begin{array}{l}\text { Social media is a valuable tool recruiters can use to } \\
\text { find the best employees }\end{array}$ & 2.97 & 3.27 & .000 \\
\hline $\begin{array}{l}\text { Employers should ask permission before performing } \\
\text { any social media searches on a potential hire }\end{array}$ & 2.23 & 3.27 & .000 \\
\hline $\begin{array}{l}\text { An individual's social media content can indicate } \\
\text { job performance }\end{array}$ & 3.25 & 2.73 & .000 \\
\hline
\end{tabular}

$1=$ Strongly Agree and $5=$ Strongly Disagree

\section{Conclusions}

Until there is more agreement between the appropriateness of certain types of posts on social media, both recruiters and those seeking employment will be at a disadvantage. Job seekers may not receive offers for positions for which they are qualified and employers may not be choosing hires from the best pool possible. Further research is needed to determine how large the impact is with inappropriate social media posts and successful job search. Differences in social media post evaluation due to industry affiliation, position being filled, and other organization characteristics should be examined.

Colleges seeking to improve their graduates' chances of placement, should, perhaps, evaluate the benefit from additional training beyond resume workshops and practice interviews. While students are likely aware their online identities can be, and probably are, being viewed, they may not know the extent to which their on-line presence is being used for evaluation and job fit, or how the recruiters perception may differ from their own with regard to a social media post. 


\section{References}

Black, S. L. \& Johnson, A. F. (2012) Employers' use of social networking sites in the selection process. Journal of Social Media in Society. 1 (1). p. 8-28.

Bohnhert, D.B.S. \& Ross, W. H. (2010) The influence of social networking web sites on the evaluation of job candidates. Cyberpsychology, Behavior and Social Networking. 13 (3). p. 341-347.

Brown, V. R. \& Vaughn, E. D. (2011) The writing on the (Facebook) wall: The use of social networking sites in hiring decisions. Journal of Business and Psychology. 26 (2). p. 219-225.

CareerBuilder (2009) [Online] Available from: http://www.careerbuilder.com/share/ aboutus/pressreleasesdetail. aspx?sd=6\%2F26\%2F2014\&id=pr829\&ed=12\%2F31\%2F2014. [Accessed: 9 March 2015]

CareerBuilder (2014) [Online] Available from: http://www.careerbuilder.com/share/ aboutus/pressreleasesdetail. aspx $? \mathrm{sd}=6 \% 2 \mathrm{~F} 26 \% 2 \mathrm{~F} 2014 \& \mathrm{id}=\mathrm{pr} 829 \& \mathrm{ed}=12 \% 2 \mathrm{~F} 31 \% 2 \mathrm{~F} 2014$. [Accessed: 9 March 2015]

CareerBuilder (2016) [Online] Available from: http://www.careerbuilder.com/share/ aboutus/pressreleasesdetail. aspx?ed $=12 \% 2 F 31 \% 2 F 2016 \& i d=p r 945 \& s d=4 \% 2 F 28 \% 2 F 2016$. [Accessed: 12 June 2017]

Chang, W. \& Madera, J. M. (2012) Using social network sites for selection purposes: An investigation of hospitality recruiters. Journal of Human Resources in Hospitality \& Tourism. 11. p. 183 - 196.

Davison, K. H., Maraist, C. \& Bing, M. N. (2011) Friend or foe? The promise and pitfalls of using social media networking sites for HR decisions. Journal of Business and Psychology. 26 (2). p, 153-159.

Davison, K. H. (2012). Big brother wants to "friend" you on Facebook. Industrial-Organizational Psychologist. 50 (2). p, 39-45.

Ellison, N., Heino, R. \& Gibbs, J. (2006) Managing impressions online: Self-preservation processes in the online dating environment. Journal of Computer-Mediated Communication. 11. p. 415-441.

El Ouirdi, M., Pais, I., Segers, J. \& El Ouirdi, A. (2016). The relationship between recruiter characteristics and applicant assessment on social media. Computers in Human Behavior. 62 (September). p. 415-422. [Online] Available from:

https://doi.org/10.1016/j.chb.2016.04.012. [Accessed: 12 June 2017]

French, A. M. \& Read, A. (2013) My mom's on Facebook: An evaluation of information sharing depth in social networking. Behaviour \& Information Technology. 32 (10). p. 1049-1059.

Goodmon, L. B., Smith, P. L., Ivancevich,D. \& Lundberg, S. (2014) Actions speak louder than personality: Effects of Facebook content on personality perceptions. North American Journal of Psychology. 16 (1). p. 105-120.

Herbold, J. \& Douma, B. (2013). Students' use of social media for job seeking: A recruitment guide for accounting firms. The CPA Journal. 68-71.

Jobvite (2014) [Online] Available from: http://www.jobvite.com/wp-content/ uploads/ 2014/10/Jobvite SocialRecruiting_Survey2014.pdf. [Accessed: 9 March 2015]

Jones, C. \& Behling, S. (2010) Uncharted waters: Using social networks in hiring decisions. Issues in Information 
Systems. 11 (1). p. 589-595.

Karl, K., Peluchette, J. \& Schlaegel, C. (2010) Who's posting Facebook faux pas? A cross cultural examination of personality differences. International Journal of Selection and Assessment. 18 (2). p. 174-186.

Kluemper, D. H. \& Rosen, P. (2009) Future employment selection methods: Evaluating social networking web sites. Journal of Managerial Psychology. 24 (6). p. 567-580.

Lamoureux, B. J. (2012) How social media is changing the hiring game. NACE Journal. 73 (1). p. 8-11.

Nikolaou, I. (2014) Social networking sites in job search and employee recruitment. International Journal of Selection and Assessment. 22 (2). p. 179 - 189.

Peluchette, J. \& Karl, K. (2008) Social Networking Profiles: An examination of student attitudes regarding use and appropriateness of content. Cyberpsychology and Behavior. 11. p. 95-97.

Peluchette, J. \& Karl, K. (2010) Examining students' intended image on Facebook: "What were they thinking?!" Journal of Education for Business. 85. p. 30-37.

Petrecca, L. (2011) More gads use social media to job hunt. USA Today. April 5. [Online] Available from Academic Search Premier (AN J0E330448434411) [Accessed: 28 June 2017]

Root, T. \& McKay, S. (2014). Student awareness of the se of social media screening by prospective employers. Journal of Education for Business. 89 4. p. 202-206.

Roulin, N. (2014) The influence of employers' use of social networking websites in selection, online selfpromotion, and personality on the likelihood of faux pas postings. International Journal of Selection and Assessment. 22 (1). p. $80-87$.

Segal, J. (2017) Social media use in hiring: Assessing the risks. HR Magazine. May 18, 2017. [Online] Available from: https://www.shrm.org/hr-today/news/hr-magazine/pages/0914-social-media-hiring.aspx. [Accessed: 12 June 2017]

Strehlke, C. (2010) Social networking sites: a starting point for career development practitioners. Journal of Employment Counseling. March (47). p. 38-48.

Wethington, B. L. \&. Bechtel, A. R. (2012) Alternative sources of information and the selection decision making process. Journal of Behavioral and Applied Management. 13 (2). p. 108-120.

Williams, J., Field, C, \& James, K. (2011). The effects of a social media policy on pharmacy students' Facebook security settings. American Journal of Pharmaceutical Education. 75 (9). p. 1-6.

Keywords: social networking sites, job seekers, recruiters, college students, SNS

Relevance to Marketing Educators, Researchers and Practitioners: Recruiters' and job seekers' perceptions of social media content are significantly different, as are the two groups' views regarding the appropriateness of social media use by recruiters to evaluate job seekers. Better training for college students is indicated in order to improve their chances for job placement after graduation.

\section{Track: Marketing Education}

